\title{
Rhythmic pulsing: linking ongoing brain activity with evoked responses
}

\author{
Ali Mazaheri ${ }^{1,2 *}$ and Ole Jensen ${ }^{1}$ \\ ' Donders Institute for Brain, Cognition and Behaviour, Radboud University Nijmegen, Nijmegen, Netherlands \\ 2 Center for Mind and Brain, University of California Davis, Davis, USA
}

Edited by:

Michael X. Cohen, University of

Amsterdam, Netherlands

Reviewed by:

Paul Sauseng,

University Medical Center Hamburg-

Eppendorf, Germany

Catherine Tallon-Baudry,

Universite Pierre et Marie Curie, France

*Correspondence:

Ali Mazaheri, Donders Institute for

Brain, Cognition and Behaviour.

Radboud University Nijmegen,

P. O. Box 9101, 6500 HB Nijmegen,

Netherlands.

e-mail:ali.mazah@gmail.com
The conventional assumption in human cognitive electrophysiology using EEG and MEG is that the presentation of a particular event such as visual or auditory stimuli evokes a "turning on" of additional brain activity that adds to the ongoing background activity. Averaging multiple event-locked trials is thought to result in the cancellation of the seemingly random phased ongoing activity while leaving the evoked response. However, recent work strongly challenges this conventional view and demonstrates that the ongoing activity is not averaged out due to specific non-sinusoidal properties. As a consquence, systematic modulations in ongoing activity can produce slow cortical evoked responses reflecting cognitive processing. In this review we introduce the concept of "rhythmic pulsing" to account for this specific non-sinusoidal property. We will explain how rhythmic pulsing can create slow evoked responses from a physiological perspective. We will also discuss how the notion of rhythmic pulsing provides a unifying framework linking ongoing oscillations, evoked responses and the brain's capacity to process incoming information.

Keywords: alpha oscillations, evoked responses, inhibition, amplitude asymmetry
EEG and MEG are used in neuroimaging studies to provide a real-time measure of respectively electric and magnetic fields produced by neuronal activity in the brain. The majority of EEG/ MEG research attempting to link human cognition to neuronal activity examines the neuronal events (i.e., evoked response) after the occurrence of an event. The ongoing activity (already present prior to the stimulus) is often considered irrelevant and is assumed to be "averaged out" across trials (Figure 1C). Recent work has challenged the dogma that ongoing activity can simply be averaged out across trials. The key aspect of these studies is the revelation that the ongoing activity in the frequency of $10 \mathrm{~Hz}$ (i.e., alpha band) contains a non-sinusoidal property referred to as "amplitude asymmetry" or "baseline shift" (Figure 1B) (Nikulin et al., 2007; Mazaheri and Jensen, 2008; van Dijk et al., 2010b).

The amplitude asymmetry of ongoing oscillations entails that the peaks are modulated stronger than troughs (or vice versa) (Figure 1B) irrespective of the DC offset/zero-line of the signal. Amplitude asymmetric oscillations have profound consequences for event-related averaging. For example if the amplitude of ongoing oscillations is suppressed such that only the peaks are reduced in magnitude but not the troughs, averaging across trials would result in the emergence of an evoked response with a shape that is similar to the time course of amplitude suppression of the oscillation (Figure 1D). Had the magnitudes of the peaks and troughs been symmetrically decreased, no evoked responses would have been generated. The critical prerequisite for this mechanism is the differential modulation of the peaks and troughs which we propose is caused by unidirectional primary currents in pyramidal cells producing the oscillations (to be discussed further).
Because of the presence of amplitude asymmetry, slow evoked responses can be generated from simple changes in the amplitude of ongoing alpha activity without any turning "on" of additional brain activity.

In this review we will discuss the concept of amplitude asymmetry and its implications to the brain's post-stimulus responses. Furthermore, we propose that ongoing alpha activity along with amplitude asymmetric properties can be conceived as rhythmic pulsing which produces bouts of inhibition every $100 \mathrm{~ms}$. Processing of a stimulus can only occur between two alpha pulses; this is consistent with the view that visual processing relies on discrete processing (VanRullen and Koch, 2003). Importantly we conjecture that this rhythmic inhibition only occurs when the "pulses" of alpha activity are at a sufficiently high amplitude. We will attempt to demonstrate that the rhythmic pulsing framework fits nicely with other theoretical models of alpha activity and could potentially offer a unified account of the brain's ongoing activity, discrete stimulus processing and evoked responses.

\section{ONGOING OSCILLATIONS AND EVOKED RESPONSES}

The evoked response reflects the brain's transient time-locked response to a stimulus or event. Evoked responses are calculated by averaging several trials (typically 30 to a 100) time-locked to a given stimulus or event. Subsequently the pre-stimulus activity (typically in a 100- to 200-ms interval) is subtracted from the trial average. The relationship between ongoing activity and evoked responses has been a matter of debate for several decades. There are currently three different theories which attempt to account for how evoked responses are generated: additivity, phase-resetting and the recently proposed amplitude asymmetry mechanism (also termed baseline-shift). 
The additive and phase-resetting models focus on the early evoked components. These early ERPs/ERFs (sometimes referred to as "exogenous components") are transient components that occur within the first few hundred milliseconds of stimulus presentation, and are widely believed to index the arrival of information to the cortex (Coles and Rugg, 1995). The amplitude asymmetry theory focuses on the late occurring components (often referred to as "endogenous") which emerge at least $100 \mathrm{~ms}$ after stimulus onset and are often sustained for hundred milliseconds or longer. There a numerous examples of the slow late components being modulated by various cognitive tasks and as such they can be viewed as a link between electrophysiology and cognition (Walter et al., 1964; Kutas and Hillyard, 1980; Sanquist et al., 1980; Hagoort and Brown, 2000; Soltani and Knight, 2000; Kilner et al., 2004; Vogel et al., 2005; Takashima et al., 2006; Rugg and Curran, 2007).

A
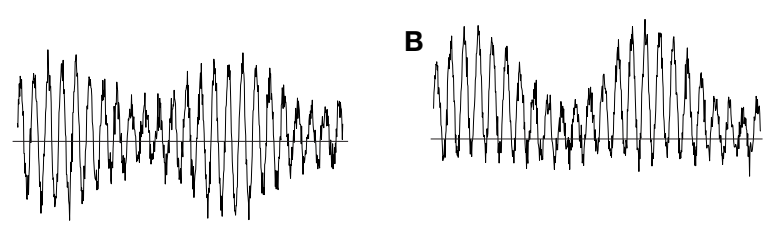

$100 \mathrm{~ms}$

C

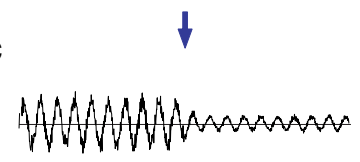

MNANAMAMmano

D
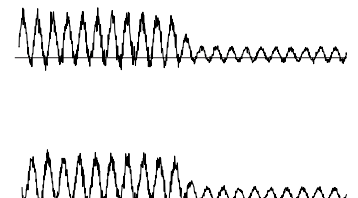
HVVWVWhamm MPMNAMAMmmam

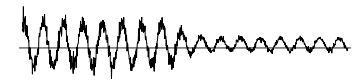

MMMMMN WVivivinamanam

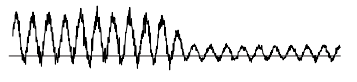

ER

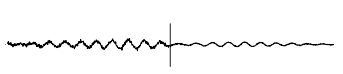

ER

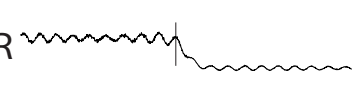

\section{ADDITIVE THEORY VERSUS PHASE-RESETTING THEORY OF EVOKED RESPONSES}

The additive theory implies that evoked and ongoing activities are separate and distinct neuronal phenomena. According to this view the stimulus "evokes" a phase-locked response adding to the activity in each trial (Figure 2A). In contrast, according to the phase-resetting theory, the phases of the ongoing background oscillations become aligned (phase-reset or partial phase-reset) to the stimulus (Figure 2B). By averaging the stimulus-locked trials, the phase-locked oscillatory activity emerges as the evoked component in the average. Since alpha oscillations $(8-12 \mathrm{~Hz})$ are the predominant ongoing activity in the EEG/MEG, it is believed that the phase-resetting of these oscillations is particularly relevant for producing the evoked activity (Makeig et al., 2002; Klimesch et al., 2004; Gruber et al., 2005).

Part of the issue in disambiguating the additive and phaseresetting theory is that the addition of a response can look much the same as a phase-reset of the oscillatory background activity. There are a number of informative and critical discussions about the merits of the phase-resetting versus additive modeling of evoked response generations (Fell et al., 2004; Shah et al., 2004; Makinen et al., 2005; Mazaheri and Jensen, 2006; Klimesch et al., 2007b, 2009; Min et al., 2007; Becker et al., 2008; Risner et al., 2009; Ritter and Becker, 2009). It is still a matter of debate how general the phase-resetting mechanism is for the generation of evoked responses.

\section{AMPLITUDE ASYMMETRY AS A MECHANISM FOR THE GENERATION OF EVOKED RESPONSES}

The amplitude fluctuations of oscillatory activity are conventionally viewed as being symmetric around zero (Figure 1A). Amplitude asymmetry refers to the observation that modulations of ongoing activity affect the peaks and the troughs of the alpha activity to different extents. This would mean that only the peak values

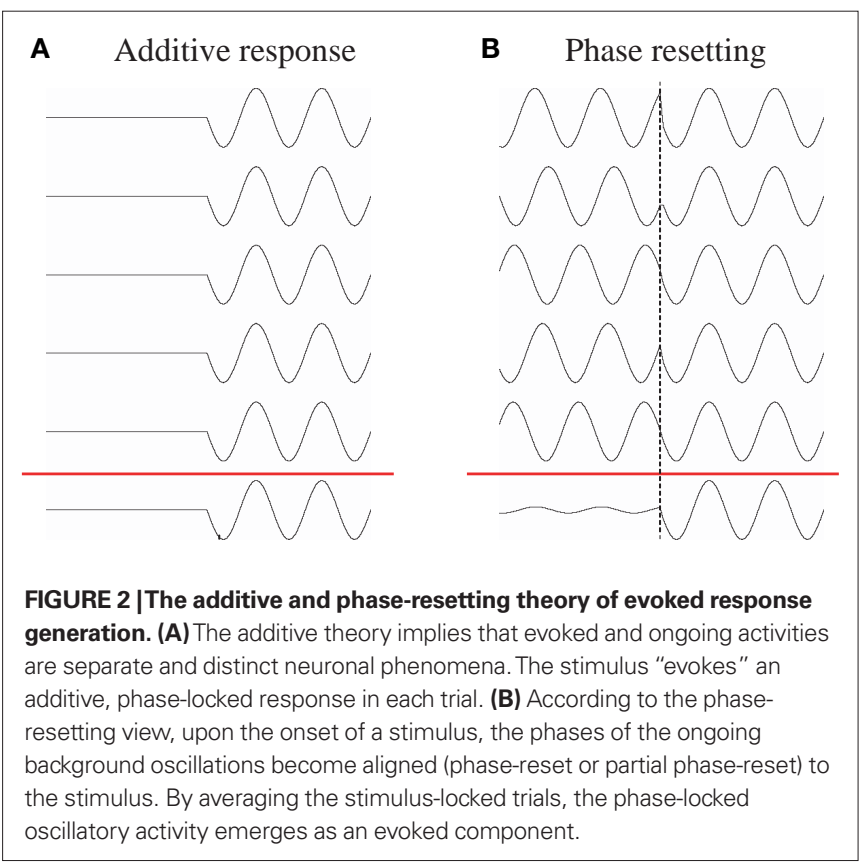


increase or decrease, while the trough values stay the same (or vice versa) (Figure 1B). Amplitude asymmetry can be found in a lot of systems around us. One way to conceptualize amplitude fluctuation asymmetry is to think of a bouncing ball: how high it jumps varies, but it cannot go lower than the floor. Another example is the light intensity in your office over days: the light at noon will vary a lot more over days compared to the light at mid-night.

One important consequence of this amplitude asymmetry of ongoing oscillations is that event-related modulations of oscillatory activity would not be "averaged out" over trials, but instead lead to the formation of slow evoked responses (Figure 1D). This is explained by a systematic depression of the peaks in response to the stimuli while the troughs remain the same. Since the phases of the individual trials are different, this will produce a slow deflection in the evoked response after trial averaging.

\section{EVIDENCE FOR THE AMPLITUDE ASYMMETRY OF OSCILLATIONS}

The amplitude asymmetry of the ongoing EEG was appreciated by Stam et al. using a measure that demonstrated that the predictability of the EEG signal in time was reduced when the signal was inverted from "peak to trough" (Stam et al., 1999b). However, the link between evoked responses and the "non-zero mean" property in oscillations was first discussed by Nikulin et al. (2007). The authors found a correlation between low frequency drifts (referred to as baseline shifts) and the $\sim 10 \mathrm{~Hz}$ somatosensory rhythm. The authors speculated that the resulting baseline shifts could play a role in the formation of somatosensory evoked responses.
A direct link between the amplitude asymmetry property of oscillations and evoked responses was empirically demonstrated by Mazaheri and Jensen (2008). In this study, a measure called the Amplitude Fluctuation Asymmetry Index $\left(\mathrm{AFA}_{\text {index }}\right)$ was first developed to quantify the asymmetry of amplitude fluctuations. The $\mathrm{AFA}_{\text {index }}$ compares the variance of the peaks with the variance of the troughs by considering the normalized difference between the two measures. Later we will elaborate on the details of this measure. Using this AFA $_{\text {index }}$ Mazaheri and Jensen (2008) were able to show that in ongoing posterior alpha activity the peaks and the troughs were indeed modulated differently. More importantly, it was shown that the direction (i.e., stronger modulation of peaks than troughs or vice versa) and magnitude of the $\mathrm{AFA}_{\text {index }}$ during a rest condition correlated with respectively the amplitude and polarity of slow ERFs in response to simple visual stimuli. Thus this study provided strong support for the notion that systematic modulations of oscillatory activity with amplitude asymmetry can produce slow evoked responses.

Recently, van Dijk et al. (2010b) were able to extend this link by demonstrating that an evoked response modulated by a cognitive task could be explained by systematic modulations in oscillatory activity. In particular the study focused on the contralateral delayed activity (CDA) component, which is a slow evoked response typically observed in working memory tasks in which hemifield specific attention is manipulated (Vogel and Machizawa, 2004; Vogel et al., 2005; Fukuda et al., 2010). The key finding was that the emergence of the CDA could be explained by modulations in alpha activity (Figure 3). Previous studies have suggested alpha activity to be

\section{Relation between the CDA, alpha power and amplitude asymmetry}

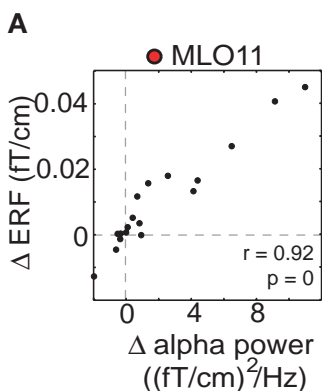

Left stim - Right stim

B
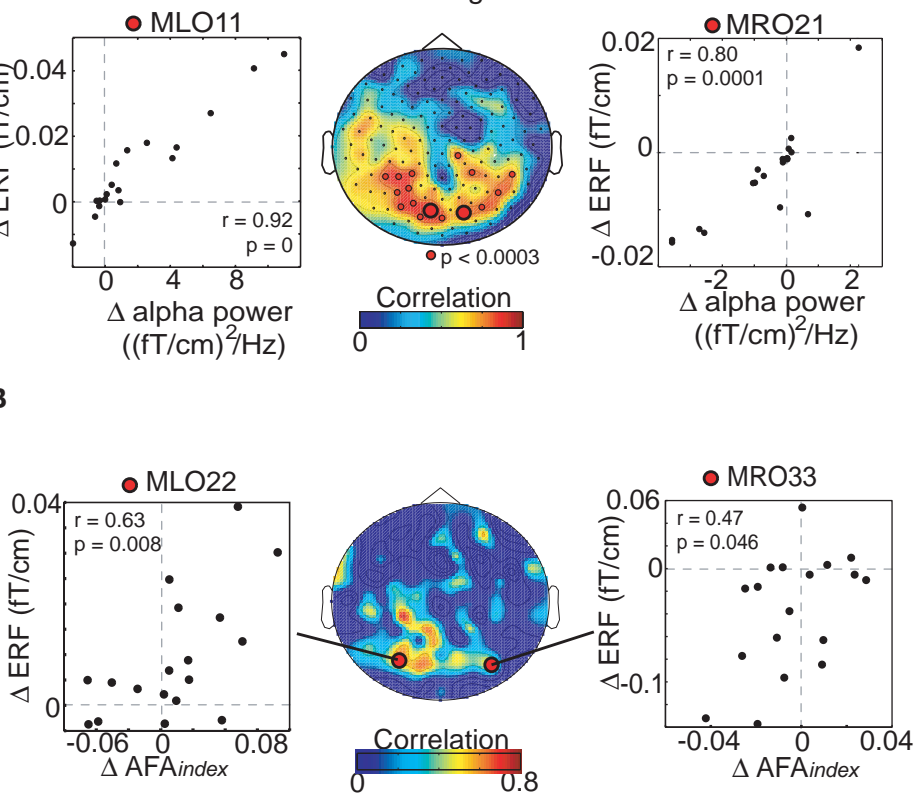

FIGURE 3 | Relation between the evoked component produced in a working memory task and modulations in hemispheric alpha power lateralization. (A) Correlation over subjects of differences in ERFs and alpha power modulations [left minus right target stimuli (stim)] for a left sensor and a right sensor (Left and Right, respectively). The correlations were highly significant. The topography (Middle) of the correlation coefficients. (B) Correlations over subjects between the differences (left minus right stimuli) in sustained ERFs and the differences in absolute alpha amplitude asymmetry (AFA index $_{\text {) }}$ during retention. Adapted from van Dijk et al. (2010b) 
involved in the functional inhibition of task-irrelevant regions (Klimesch, 1999; Klimesch et al., 1999, 2007a; Jensen et al., 2002; Jokisch and Jensen, 2007; Tuladhar et al., 2007; Jensen and Mazaheri, in press). Thus van Dijk et al. (2010b) proposed that the CDA is not attributable to an additive process reflecting memory maintenance per se but rather to changes in ongoing oscillatory alpha activity reflecting inhibition of task-irrelevant regions, while routing information to task-relevant regions. This view is further supported by recent findings of Sauseng et al. (2009) in which a similar design was used to demonstrate that modulation of hemispheric alpha lateralization predicted memory capacity based on efficient suppression of irrelevant information in short-term memory.

Although the amplitude asymmetry model suggests that certain endogenous responses are produced by modulations in oscillatory power, this does not question the merits of previous ERP/ERF studies. If a specific slow ERP/ERF is revealed to be produced by modulations in oscillatory activity, this does not mean that the conventional ERP/ERF analysis is inappropriate as a tool in cognitive neuroscience. Rather, if the mechanism of a particular evoked response can be linked to a modulation of ongoing activity, it could provide a stronger account for the neuronal substrate generating the slow evoked responses. Also, it can help interpreting the functional role of the evoked responses given that one can build on the insight gained from the role of oscillatory activity (see, e.g., van Dijk et al., 2010b).

\section{PREREOUISITE FOR LINKING THE MODULATION OF ONGOING OSCILLATIONS TO EVOKED COMPONENTS}

How is it possible to determine if the mechanism behind a specific evoked response is due to modulation of ongoing activity with amplitude asymmetry? We introduce four prerequisites for linking modulations of oscillatory activity to evoked component generation.

1. The ongoing MEG/EEG oscillations must be modulated in amplitude by the stimuli or event

2. This amplitude modulation of the ongoing activity must correlate with the time course of the evoked response (over trials or subjects)

3. The ongoing oscillations must have an amplitude asymmetry

4. The magnitude and/or polarity of the amplitude asymmetry must relate to the amplitude and/or polarity of the evoked responses (over trials or subjects)

Event-related changes in oscillatory alpha activity have been demonstrated in many cognitive paradigms (Klimesch et al., 1997; Salenius et al., 1997; Klimesch, 1999; Jensen et al., 2002; Makeig et al., 2004; Mazaheri and Picton, 2005; Bastiaansen and Hagoort, 2006; Sauseng et al., 2006; Jokisch and Jensen, 2007). Perhaps the most challenging step in linking oscillatory changes to evoked responses is the quantification of the amplitude asymmetry of the ongoing signal. For this step, two different measures have so far been proposed: the $\mathrm{AFA}_{\text {index }}$ (Mazaheri and Jensen, 2008) and baseline shift index (BSI) (Nikulin et al., 2007).

\section{ANALYTICAL METHODS FOR MEASURING AMPLITUDE ASYMMETRY}

The AFA $_{\text {index }}$ quantifies the ratio of the variance of the peaks and troughs where $S_{\text {peaks }}$ and $S_{\text {troughs }}$ refer to the peak and trough values identified in the ongoing oscillatory signal:
$\operatorname{AFA}_{\text {index }}=\frac{\operatorname{Var}\left(S_{\text {peaks }}\right)-\operatorname{Var}\left(S_{\text {troughs }}\right)}{\operatorname{Var}\left(S_{\text {peaks }}\right)+\operatorname{Var}\left(S_{\text {troughs }}\right)}$

When using the $\mathrm{AFA}_{\text {index }}$, the signal is first bandpass-filtered in the frequency band of interest (Figure 4A). Next the time points for the peaks and troughs of the bandpassed data can be identified. These time points are then used to obtain the signal values of peaks and troughs in the raw data. The variance of these values is then used to calculate the $\mathrm{AFA}_{\text {index: }}$.

Accordingly, positive $\mathrm{AFA}_{\text {index }}$ values indicate a stronger modulation of the peaks and negative values indicate a stronger modulation of the troughs, while values close to zero indicate symmetrical modulation.

By considering the difference between the variance of the peaks and troughs to the ratio of amplitudes, this measure is relatively immune to DC-like offsets that sometimes appear in MEG and EEG measurements. In order to ensure that this measure was not a consequence of a slow DC offset interacting with the alpha rhythm we (Mazaheri and Jensen, 2008) investigated various principles of actions using constructed surrogate signals. These simulations demonstrated that the $\mathrm{AFA}_{\text {index }}$ successfully can detect true asymmetric amplitude fluctuation while being immune to slow multiplicative or additive modulations. The simulations can be seen in Figure 5.

When applying the $\mathrm{AFA}_{\text {index }}$ one issue to consider is the time window in which the variance across peaks and troughs are measured. This time window would be dependent on the frequency of interest. A very short time window is not optimal, since then there simply would not be enough peaks/troughs to reliably assess the variance. To quantity the amplitude asymmetry of alpha and beta oscillations Mazaheri and Jensen (2008) used a time window of $5 \mathrm{~s}$ to calculate the asymmetry index, whereas van Dijk et al. (2010b) was able to successfully do this using a period of $1 \mathrm{~s}$. However, future empirical work needs to be done to precisely characterize the optimal temporal parameters relevant for detection of amplitude asymmetry.

It should be mentioned that the $\mathrm{AFA}_{\text {index }}$ can potentially be sensitive to harmonics present in the signal (Nikulin et al., 2010). For instance, $20 \mathrm{~Hz}$ harmonics can in some cases produce a non-zero $\mathrm{AFA}_{\text {index }}$ in the $10 \mathrm{~Hz}$ band. However, a non-zero $\mathrm{AFA}_{\text {index }}$ due to harmonics cannot produce slow evoked responses (Jensen et al., 2010; Nikulin et al., 2010) and as such would not have a relationship to the amplitude and polarity of evoked responses. One way to reduce the effect of harmonics on the $\mathrm{AFA}_{\text {index }}$ is to lowpass filter the raw data at the frequency below the harmonic but above the frequency of interest (in the case of the alpha band it would be $\sim 15 \mathrm{~Hz}$ ). Importantly the $\mathrm{AFA}_{\text {index }}$ can be correlated with both magnitude and/or the polarity of evoked responses (Figures 3 and 6). This has been successfully done for both visual evoked responses and working memory related evoked responses (Mazaheri and Jensen, 2008; van Dijk et al., 2010b). Thus, given that the $\mathrm{AFA}_{\text {index }}$ was strongly correlated with the evoked responses, these findings cannot be explained by harmonics of the alpha oscillation.

The BSI is an alternative measure to the $\mathrm{AFA}_{\text {index }}$ and quantifies the correlation between oscillatory activity in a given frequency band and low frequency fluctuations (Figure 7). First, the ongoing activity is filtered in two ways: using bandpass filters (e.g., 8-12 Hz) and a lowpass filter at $3 \mathrm{~Hz}$. The BSI reflects the regression between the bandpassed and lowpass filtered signal's amplitude values 
A

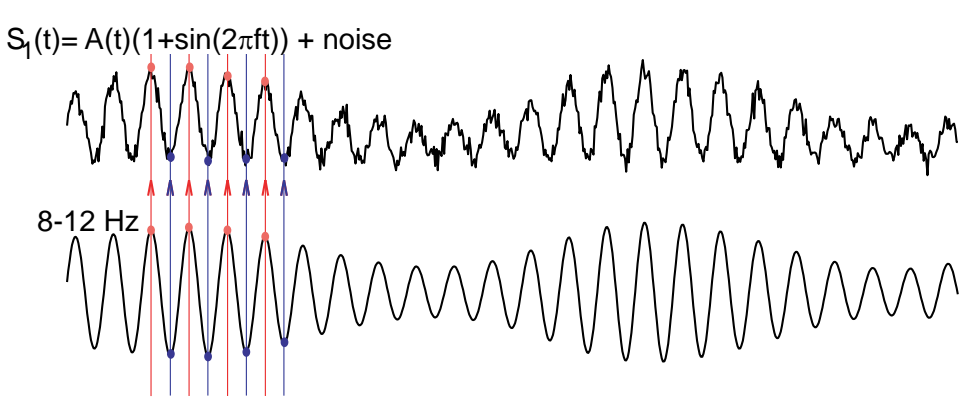

$S_{1}(t)=A(t)(1+\sin (2 \pi f t))+$ noise

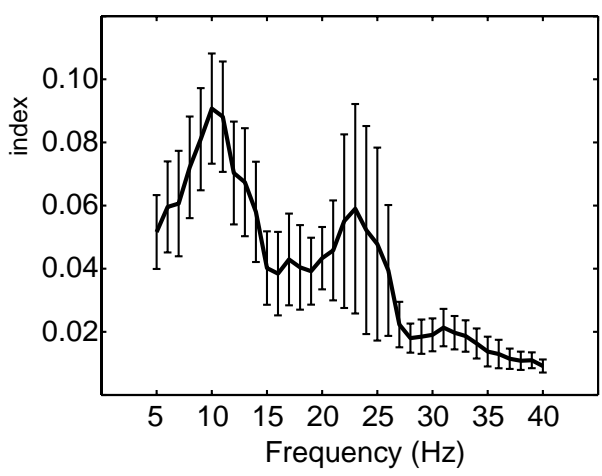

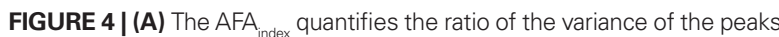
(red dots) versus the variance of the troughs (blue dots) identified in the ongoing oscillatory signal. The signal is first bandpass-filtered in the frequency band of interest. Next the time points for the peaks and troughs of the bandpassed data can be identified. These time points can then be

(Nikulin et al., 2007). The measure does depend on extensive preprocessing and it would be highly interesting to investigate if it has a quantitative relationship with evoked responses.

In conclusion amplitude asymmetry has been demonstrated to be an intrinsic property of ongoing oscillatory activity in the alpha band using different analytic techniques. Moreover a strong linkbetween the amplitude asymmetry of alpha activity and evoked responses has also been established. We propose that amplitude asymmetric oscillations can be viewed as rhythmic pulsing through which information processing is facilitated or inhibited. In the next section we will discuss the hypothesized underlying physiology of amplitude asymmetric alpha activity and its function in rhythmic pulsing.

\section{THE UNDERLYING PHYSIOLOGY OF RHYTHMIC PULSING}

Which physiological mechanisms can account for amplitude asymmetry? EEG and MEG signals are primarily thought to be produced by dendritic currents in pyramidal cells (Hamalainen et al., 1993). These intracellular currents are typically generated by electrical events at the apical dendrite or the soma. Such events include excitatory synaptic input at distal dendrites and after-hyperpolarization currents (Wu and Okada, 1999; Murakami and Okada, 2006). In order to generate oscillatory activity with symmetric amplitude fluctuations (Figure 1A) there must be intracellular currents propagating forward and backward down the dendrites with the same magnitude (respectively producing the troughs and peaks).

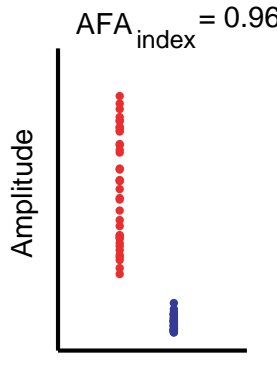

used to obtain the signal values of peaks and troughs in the raw data. (B) The AFA $_{\text {index }}$ can be used to calculated amplitude asymmetry spectra of spontaneous MEG data during rest. Note that the amplitude asymmetry is constrained to the alpha and beta band. Adapted from Mazaheri and Jensen (2008).

However, even though back-propagating dendrite currents are known to exist, it would be unlikely that they exactly match the synaptic forward propagating currents. We conjecture that the currents producing the alpha activity are asymmetric, i.e., primarily explained by forward propagating dendritic currents most likely due to excitatory synaptic inputs and after-hyperpolarization currents. It is this asymmetry between the magnitude of forward and backwards current flow that gives the ongoing alpha activity its amplitude asymmetry property (Figures $\mathbf{1 B}$ and $\mathbf{8 B}$ ). It should be mentioned that these primary intracellular dendritic currents produce instantaneous return currents (also known as volume currents). While MEG primarily detects the magnetic fields produced by the intracellular dendritic currents, EEG detects the differences in scalp potentials arising from the return currents. An illustration of this proposed neurobiological mechanism of asymmetry and its implications for MEG and EEG measurements can be seen in Figure 8.

We postulate that the amplitude asymmetric alpha activity can be viewed as rhythmic pulses producing bouts of inhibition repeated every $100 \mathrm{~ms}$. How might this inhibition come about? GABAergic feedbacks from interneurons have been strongly implicated in the physiological mechanism generating the alpha rhythm (Lopes da Silva et al., 1976; Crunelli and Leresche, 1991; Jones et al., 2000). Thus it is possible that rhythmic neuronal activity generating the alpha oscillations is a consequence of a GABAergic inhibitory feedback paced by neocortical or thalamic rhythm generators (Hughes and Crunelli, 2005; Lorincz et al., 2008, 2009). This 


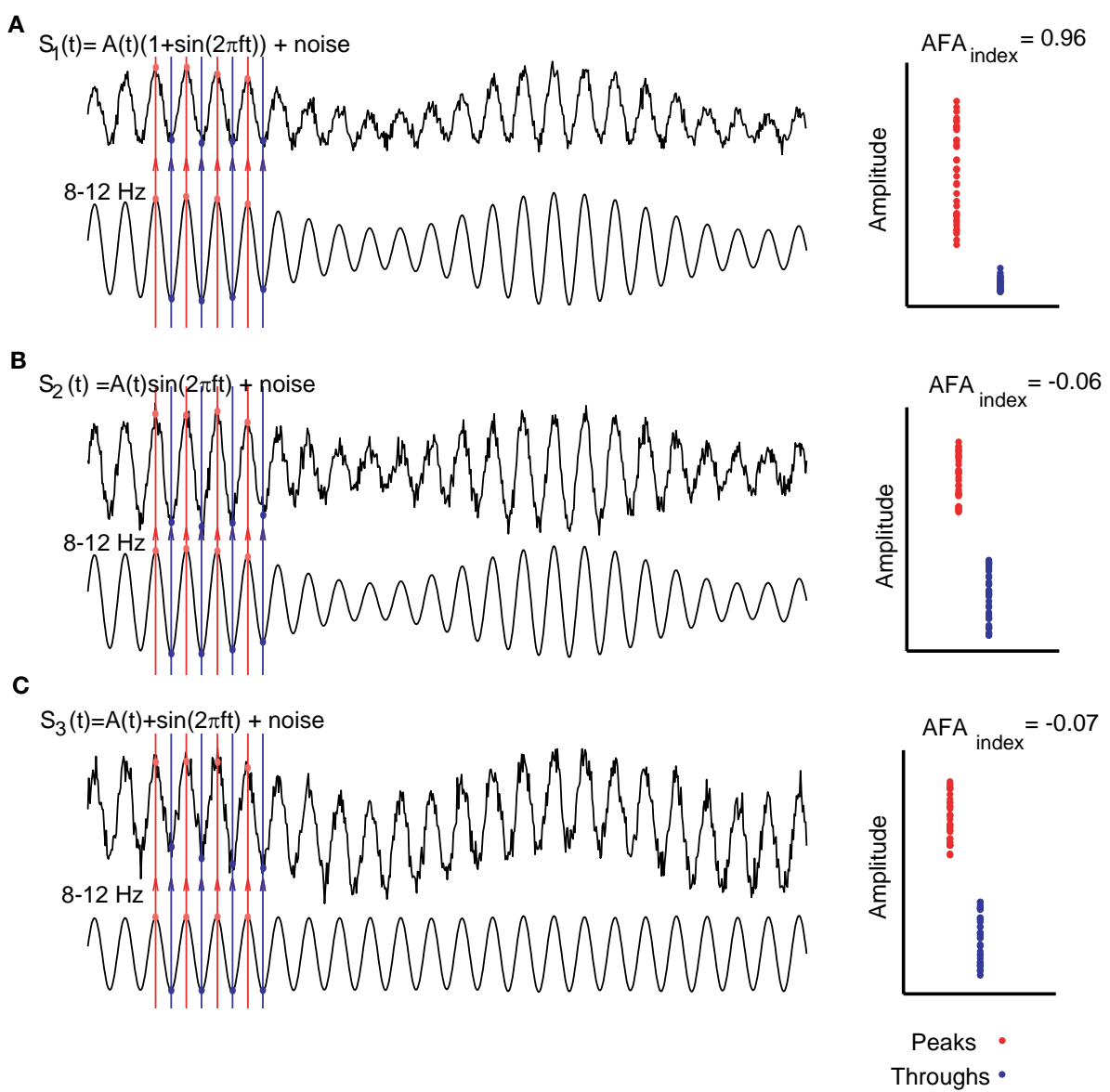

FIGURE 5 | Various simulations in which surrogate signals were used to test the AFA $_{\text {index }}$ (A) The signal, $s_{1}(t)$, was designed to have an amplitude asymmetry. The amplitude modulation was determined by a slower signal A(t). Clearly the peaks (red dots) are more modulated than the troughs (blue dots) yielding a strong AFA ${ }_{\text {index }}$ (B) The signal, $s_{2}(t)$, was designed such that the slow modulations,
$A(t)$, affected the alpha rhythm in a multiplicative manner. Thus peaks and troughs

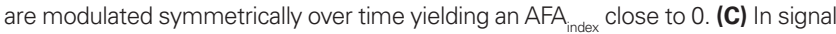
$\mathrm{S}_{3}(\mathrm{t})$ slow modulations added to the alpha oscillations (DC-like offset of the signal). This affected peaks and troughs in the same direction producing an AFA $_{\text {index }}$ close to 0. Adapted from Mazaheri and Jensen (2008).

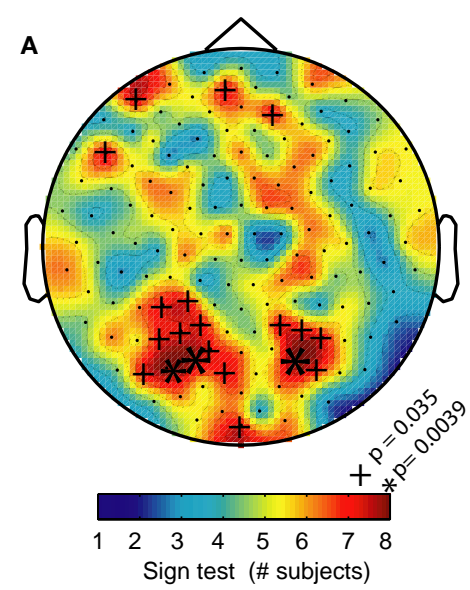

FIGURE 6 |The AFA $_{\text {index }}$ can used to predict the polarity and magnitude of evoked responses. (A) The consistency between the sign of the AFA (from eyes closed data) and the sign of the modulation in slow visually evoked ERF with alpha power. The color code represents the number of consistent signs over the eight subjects. More than six consistent signs are considered

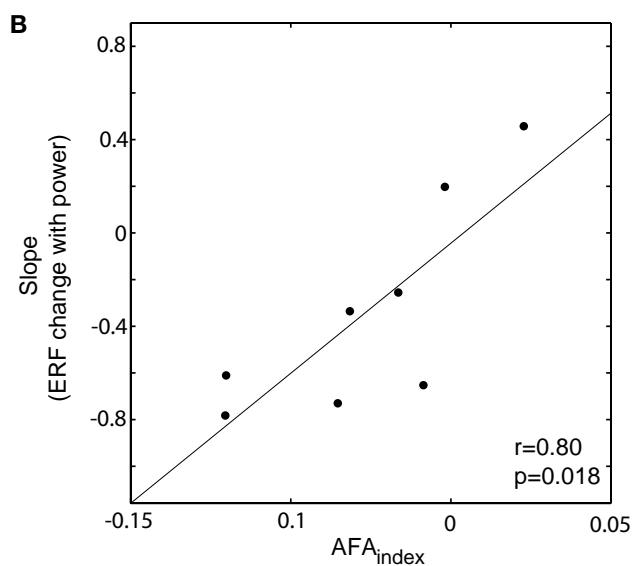

significant (binomial test). (B) The correlation between the AFA $_{\text {index }}$ and the slope of slow ERF modulation with alpha power. The high correlation strongly suggests that the slow modulations in the ERFs are produced by changes in asymmetric amplitude changes of alpha power. Adapted from Mazaheri and Jensen (2008). 

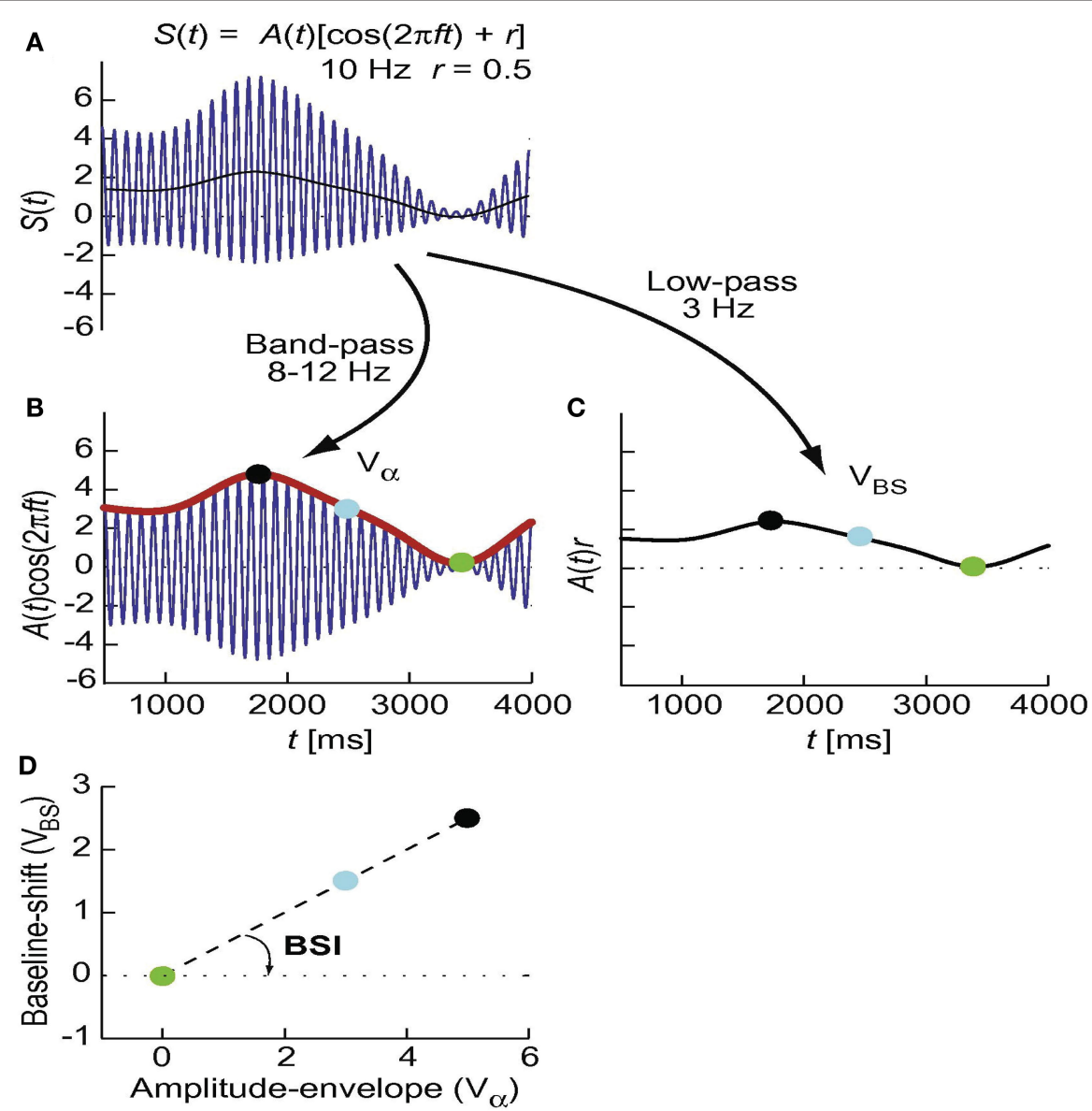

FIGURE 7 | Schematic presentation of the derivation of the baseline shift index (BSI). First, the signal in (A) is filtered in two ways to separate the amplitude envelope of the oscillations and the baseline shift $A(t) r$ [thin black line in (A)]. The amplitude envelope $\left(\mathrm{V}_{\alpha}\right)$ is obtained using bandpass filters (e.g., 8-12 Hz) and the Hilbert transform [(B), red thick line]. The baseline shifts $\left(V_{B S}\right)$ are part of the signal at considerably lower frequencies than the oscillatory "carrier" signal and, therefore, are obtained by lowpass filtering,

GABAergic feedback could serve to directly silence processing in pyramidal neurons or reduce the efficacy of excitatory input by shunting inhibition.

In order to further explain how pulsed inhibition could be reflected in the neuronal firing of pyramidal cells and subsequently the MEG signal, we constructed a simple model (Figure 9). In this model, 500 pyramidal cells are modulated by a $10 \mathrm{~Hz}$ inhibitory signal. The signal serves to silence the firing of the pyramidal cells in a phasic manner. As a consequence a pattern of rhythmic pulsing emerges. The MEG signal is thought to be produced by the dendritic current in the pyramidal cells. Thus the inhibition results in an MEG signal having amplitude asymmetry. This model might also explain the inverse relationship between the alpha activity and the BOLD signal (Goldman et al., 2002; Goncalves et al., 2006; Laufs et al., 2006; Ritter et al., 2009; Scheeringa et al., 2009; Yuan et al., 2010). In the example in Figure 9, the firing rate is highest (e.g., $0.4-0.6 \mathrm{~s}$ ) when the "10 Hz signal" is low compared to when the " $10 \mathrm{~Hz}$ signal" is high (e.g., $0.8-1.0 \mathrm{~s}$ ). Since firing rate is linked to the BOLD signal, it would have a negative relationship to the alpha activity. e.g., at $3 \mathrm{~Hz}(\mathbf{C})$. The amplitude-envelope values are sorted into bins (e.g., 20 bins) and values from the baseline shift signal are sorted into the corresponding bins (note the temporal correspondence of dots with the same color). (D) Within-bin averaged values for the amplitude envelope and baseline shifts are plotted against each other and the slope of the regression line is calculated. This slope is the baseline shift index or BSI. Adapted from Nikulin et al. (2007).

Interestingly, amplitude asymmetry can emerge from even very simple models of oscillatory activity. In 1976, Lopes da Silva et al. proposed a computational model that could account for certain features of the alpha activity. The model was composed of thalamocortical relay neurons coupled with inhibitory interneurons (Lopes da Silva et al., 1976; Stam et al., 1999a). As seen in Figure 10, this model produced alpha oscillations with amplitude asymmetry (even though this was not essential for the authors). This underscores that amplitude asymmetry is a natural phenomenon that easily can arise from physiological models of oscillatory activity. In fact, we posit that amplitude asymmetry is the norm and amplitude symmetry is the exception.

\section{EVIDENCE SUPPORTING RHYTHMIC PULSING CROSS-FREOUENCY PHASE-AMPLITUDE COUPLING}

The rhythmic pulsing view fits conceptually well with recent evidence that the power of gamma oscillations is phase-locked to posterior alpha activity. Gamma oscillations have long been implicated in neuronal processing in various tasks (Fell et al., 2003; 
A

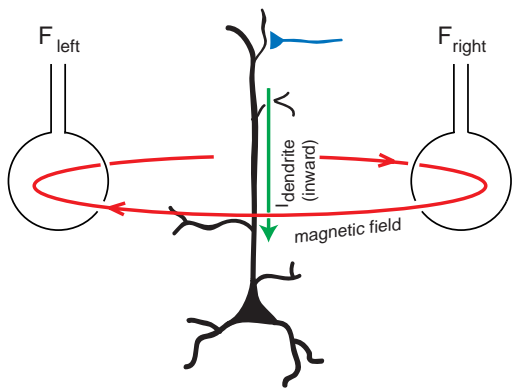

MEG

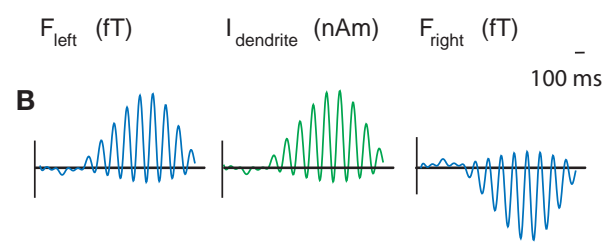

C

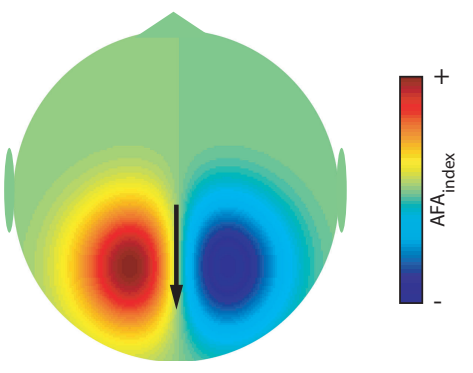

FIGURE 8 | (A) MEG signals measured outside the human scalp are primarily thought to be produced by magnetic fields induced by dendritic intracellular currents $\left(I_{\text {dendrite }}\right)$ in pyramidal cells. EEG detects the differences in scalp potentials arising from the return currents. The polarity of the field will be determined by the direction (inward or outward) of the currents in the dendrites. (B) We propose
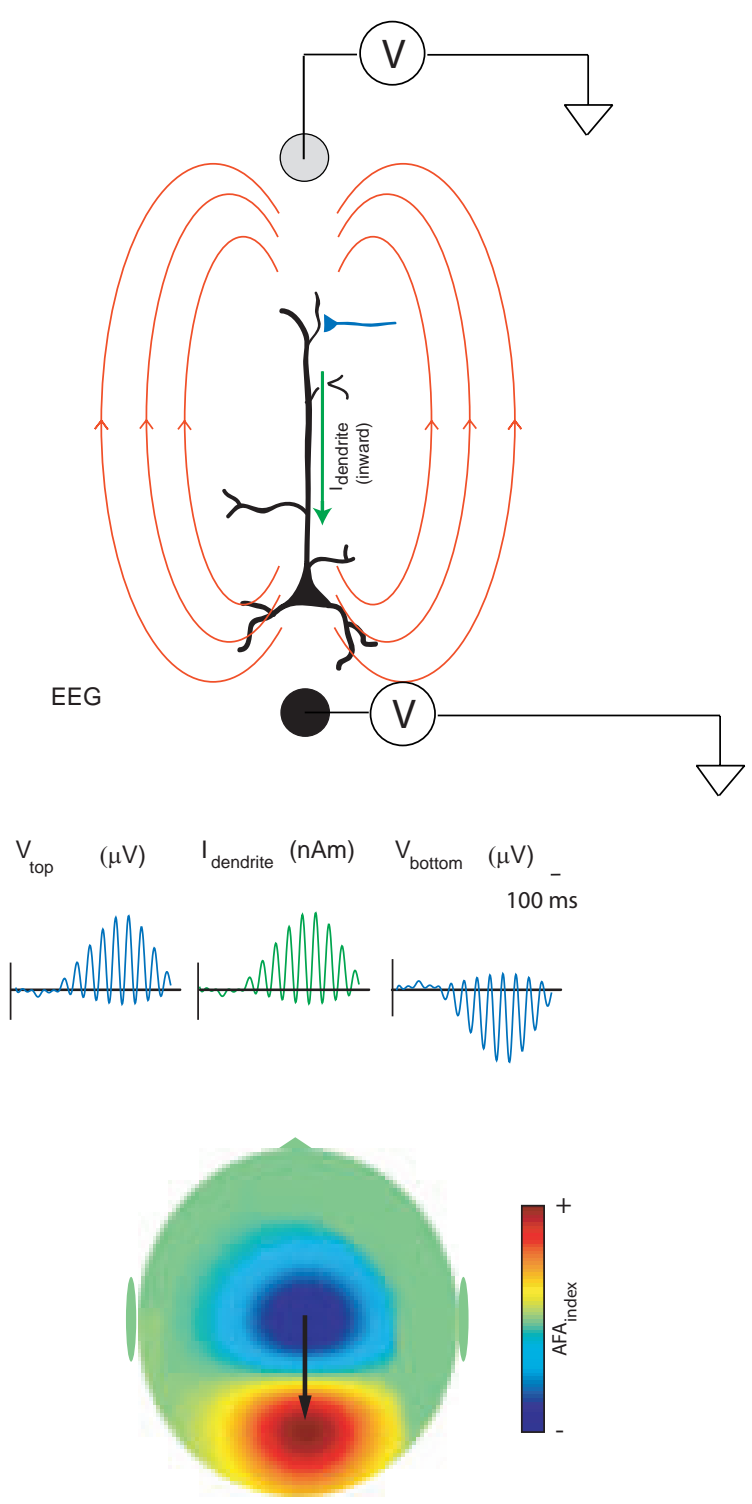

that the amplitude modulations of the oscillatory activity are asymmetric such that the peaks are modulated stronger than the troughs. For the $10 \mathrm{~Hz}$ alpha activity this is explained by bouts of inward dendritic currents every $100 \mathrm{~ms}$. (C) The topography of the amplitude asymmetry of the MEG fields and EEG potentials. Adapted and modified from Mazaheri and Jensen (2008).

coupling across oscillations, it is not necessary for oscillations to be amplitude asymmetric to exhibit phase-power relationships. Moreover a direct a functional link between alpha phase and gamma power still remains to be empirically determined.

\section{ONGOING ALPHA AMPLITUDE AND INHIBITION}

Klimesch et al. (2007a) recently proposed the inhibition-timing hypothesis where alpha oscillations play an important role in the brain's capacity to process information. They postulated that a reduction in the amplitude of the ongoing alpha activity reflects a state of comparatively high excitability, whereas high amplitudes reflect a state of inhibition (comparatively low excitability). 


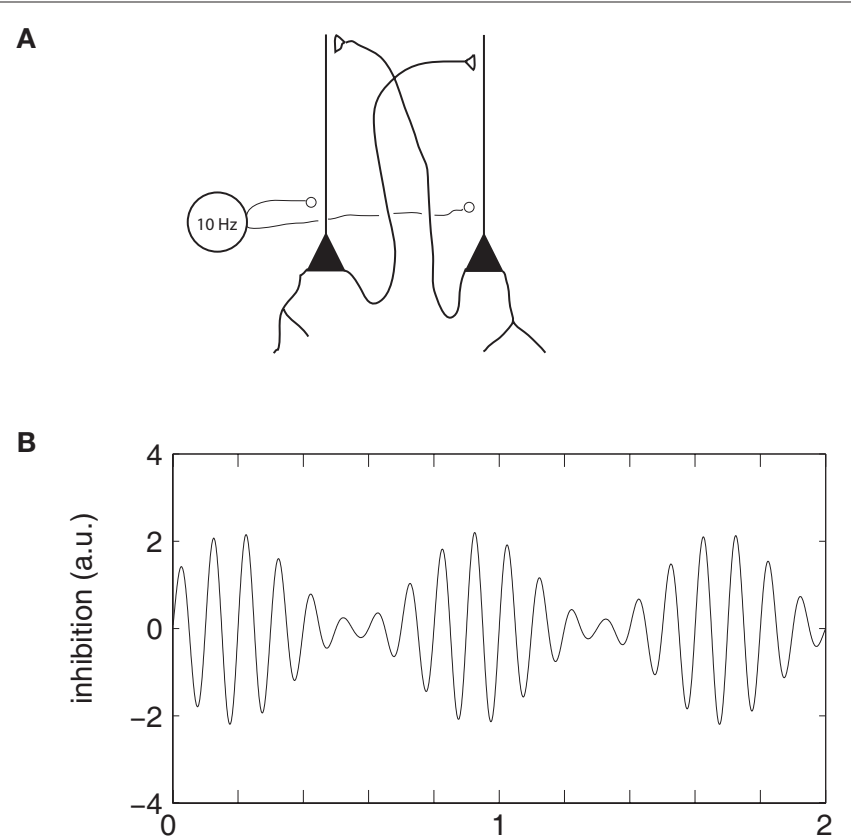

C

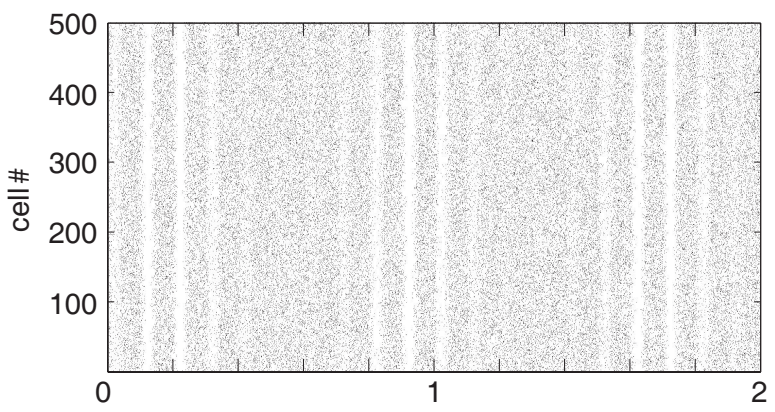

D

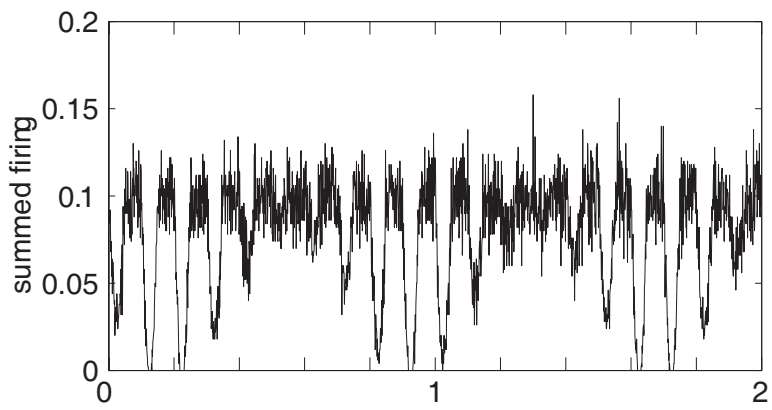

E

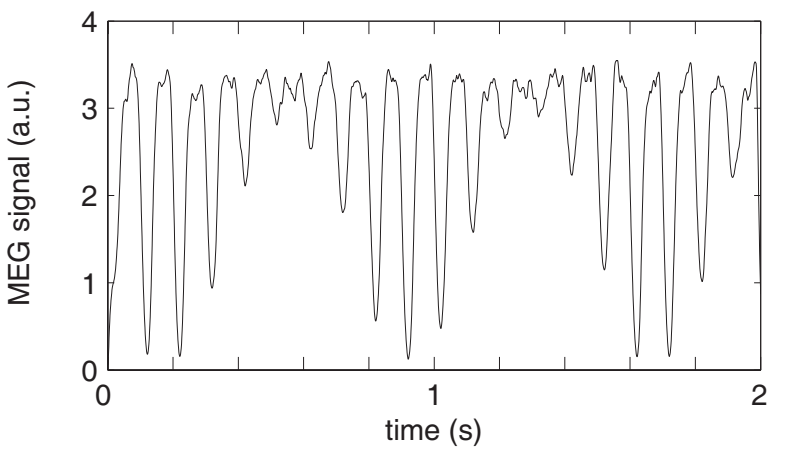

In fact it was first suggested almost 80 years ago that that the cortex exhibits cyclic changes between maximal and minimal responsiveness (Bishop, 1933). Since then a number of studies have showed an influence of the phase of the ongoing alpha activity on processing of visual stimuli (Callaway and Yeager, 1960; Varela et al., 1981). Two very recent EEG studies by Mathewson et al. (2009) and Busch et al. (2009) have reported a functional link between the phase of the pre-stimulus alpha oscillations and conscious perception by using threshold stimuli. The authors conjecture that the alpha inhibition is limited to parts of the cycle, generating a form of "pulsed inhibition." We suggest that this pulsed inhibition occurs as a function of the amplitude asymmetric property of the ongoing oscillations.

Evidence supporting the inhibitory nature of alpha activity has been found in a number of studies that demonstrate an increase or decrease in the amplitude of alpha activity depending on the visual stream engaged in a given task. (Worden et al., 2000; Thut et al., 2003, 2006; Kelly, et al., 2006; Jokisch and Jensen, 2007; Medendorp et al., 2007; Rihs et al., 2007; Romei et al., 2007, 2008; van Dijk et al., 2008; Zhang et al., 2008). The functional role of alpha in defining the brain state does not seem to be restricted to visual tasks. A recent study has found alpha power lateralization at parieto-occipital sites to be modulated by the direction of auditory attention to continuous speech in space (Kerlin et al., 2010). In a working memory study on maintaining pitches, alpha activity from left superior temporal areas increased during the retention interval (van Dijk et al., 2010a). This temporal alpha activity, possibly being the taurhythm (Lehtela et al., 1997) is likely to reflect inhibition of the left auditory cortex in order to allocate resources to the right auditory cortex involved in pitch processing. In a somatosensory working memory task, the alpha activity decreased in the primary sensorimotor cortex contralateral to the engaged hand while it increased in the ipsilateral hemisphere (Haegens et al., 2010). In summary these studies are consistent with the idea that the functional inhibition of a region in the cortex is mediated by increasing oscillatory activity in the alpha band (8-13 $\mathrm{Hz}$ ) (Jensen and Mazaheri, in press).

FIGURE 9 |A simple model explaining how pulsed inhibition is reflected in neuronal firing of pyramidal cells and subsequently the MEG signal. (A) Pyramidal cells are mutually synaptically coupled. The synaptic currents produce the MEG signal. We here assume a Poisson distribution of firing which is phasically silenced by an inhibitory GABAergic signal. (B) The signal at $10 \mathrm{~Hz}$ exercises an inhibitory drive silencing the firing of the pyramidal cells in a phasic manner. The magnitude of the inhibition is modulated by an arbitrary slower signal. (C) A raster diagram showing the firing of 500 pyramidal cells. The bouts of inhibition serve to silence the firing. In periods when the inhibition is low, the firing will persist (e.g., 0.4-0.6 s). (D) The summed firing of all the pyramidal cells (summed using a $\Delta t=1 \mathrm{~ms}$ sliding time window). (E) Each spike was convolved with an alpha-function $\left(\alpha(t)=(t / \tau) \mathrm{e}^{1-(t / \tau)}\right.$, where $\left.\tau=5 \mathrm{~ms}\right)$ in order to approximate the post-synaptic current. These post-synaptic currents were then averaged over the pyramidal neurons. The "MEG signal" (arbitrary units, arbitrary offset) is proportional to the total dendritic current. Note that the inhibition results in a rhythmically pulsed MEG signal with amplitude asymmetry. 


\section{Alpha Model}

$\mu \mathrm{V}$

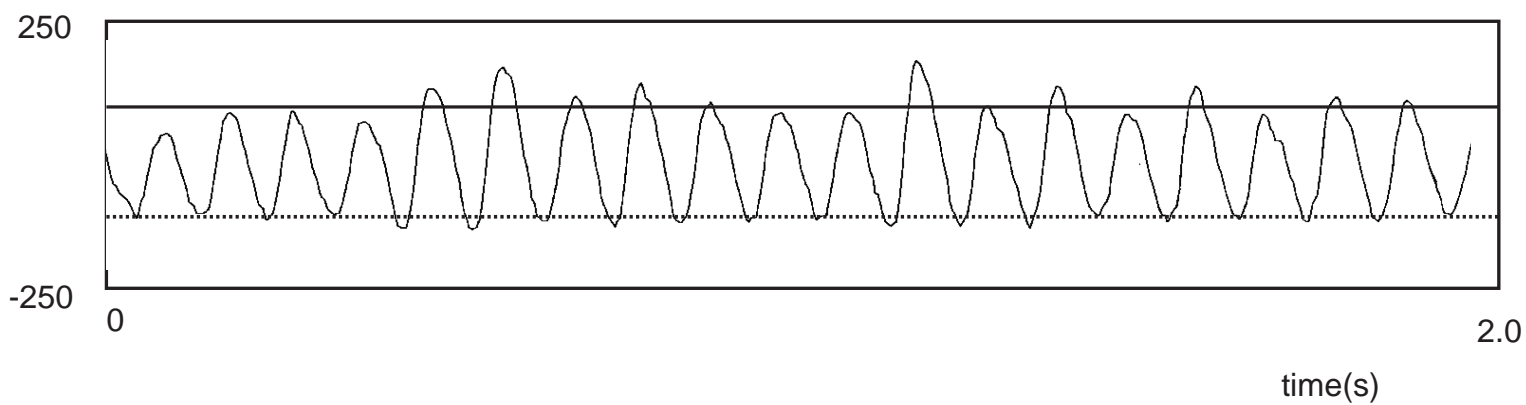

FIGURE 10 |An alpha rhythm time-series displaying non-sinusoidal properties. Prior to the discovery of its link to the formation of evoked responses, amplitude asymmetry had been observed in models designed to produce alpha activity. Here is an example adapted from Stam et al. (1999a).

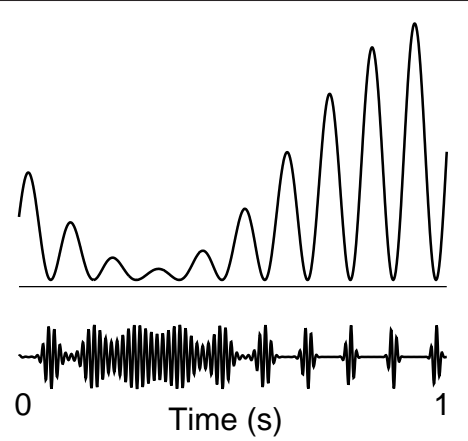

FIGURE 11 | Rhythmic pulsing and it implications for processing - a hypothesis. The gamma burst (e.g., involved in visual processing) can only occur when the alpha signal is low enough, e.g., at the troughs. Thus, the periods of gamma activity become briefer with stronger alpha. When the alpha activity is sufficiently weak, gamma can occur during the whole cycle. This scheme is consistent with the notion of rhythmic pulsing. Adapted from Osipova et al. (2008).

\section{OPEN QUESTIONS AND CAVEATS FOR FUTURE RESEARCH RELATIONSHIP BETWEEN OSCILLATIONS AND BOLD}

Understanding the relationship between the brain's ongoing oscillations and evoked activity is quite important to the field of human electrophysiology since it is bound to lead to a fundamentally better understanding of how the signals measured by MEG/ EEG link to cognition. Moreover understanding this relationship can extend beyond the realm of EEG and MEG research because evoked responses provide a critical link between the hemodynamic response measured by the fMRI and the underlying temporal dynamics of neuronal activity. There are now a number of studies that have correlated the amplitude of alpha activity with the BOLD signal (Goldman et al., 2002; Goncalves et al., 2006; Laufs et al., 2006; Ritter et al., 2009; Scheeringa et al., 2009; Yuan et al., 2010). However, the relationships between the prestimulus phase and amplitude of the ongoing alpha activity and the stimulus evoked BOLD have thus far not been investigated. If a relationship between pre-stimulus alpha phase and BOLD is demonstrated it would support the phasic aspect of the alpha oscillations and demonstrate that alpha activity has direct con- sequences for neuronal excitability. Moreover, the combination of brain stimulation by transcranial magnetic stimulation (TMS) with EEG can provide real-time information on the phasic aspects of cortical reactivity (Thut and Miniussi, 2009; Miniussi and Thut, 2010). Indeed, recent studies have found that the phase of the spinal beta rhythm in which the input (a TMS pulse) arrives modulated the gain of this input (Maki and Ilmoniemi, 2010; van Elswijk et al., 2010).

\section{WHAT ABOUT OTHER RHYTHMS?}

The mechanism behind amplitude asymmetry of oscillatory activity need not be specific to alpha oscillations. The unidirectional primary currents in pyramidal cells could also be responsible for asymmetry in other frequencies bands a well. There is a body of research pointing to delta oscillations serving a fundamental role in the active input selection at primary sensory cortex level (Lakatos et al., 2007, 2008; Schroeder and Lakatos, 2009). According to this view the phase of the delta rhythm serves as a master controller of neuronal excitability. The relationship between delta phase and alpha activity needs to be explored. For example delta activity might reflect a top-down regulations of the alpha activity. Thus the time course of delta active could be comparable to the ERPs/ERFs produced by the modulation of the amplitude asymmetric alpha activity. The late occurring slow evoked responses can be viewed as the link between electrophysiology and cognition. These responses have been found to index working memory capacity (Vogel et al., 2005), long-term memory encoding and recognition (Sanquist et al., 1980; Takashima et al., 2006; Rugg and Curran, 2007), action monitoring (Kilner et al., 2004), language comprehension (Kutas and Hillyard, 1980; Hagoort and Brown, 2000), response preparation (Walter et al., 1964), and novelty detection (Soltani and Knight, 2000). Yet the exact physiological mechanism for how these responses are generated is still unknown.

Future work is required in order to investigate if the principle of amplitude asymmetry and the generation of evoked responses can be generalized to frequency bands beyond the alpha range. Averaging epochs of amplitude asymmetric oscillations will result in an of evoked response with a shape that is similar to the time course of amplitude suppression of the oscillation. A number of 
studies have now shown that modulations in oscillatory activity at various frequency bands to occur at the same time window as cognitive evoked responses such as the Dm, P300, N400, and P600 effect (Klimesch et al., 1996; Mazaheri and Picton, 2005; Davidson and Indefrey, 2007). It would of interest to see if the mechanism of amplitude asymmetry plays a role in the formation of these responses.

If the mechanism underlying these responses is revealed to be explained by amplitude asymmetry of ongoing oscillations, it would in no way discount the value of conventional ERP/ERF analysis.
Rather this would serve to take the human electrophysiology a very significant step further into linking the oscillatory firing of neuronal populations to human cognition.

\section{ACKNOWLEDGMENTS}

This research was supported by The Netherlands Organization for Scientific Research (NWO): Rubicon Grant for AM, Innovational Research Incentive Schemes, VICI grant; ALW Open Competition, grant number 817.02.010. We would like to thank Dr. Risa Sawaki for comments on an earlier version of the manuscript.

\section{REFERENCES}

Basar, E., Basar-Eroglu, C., Karakas, S., and Schurmann, M. (2001). Gamma, alpha, delta, and theta oscillations govern cognitive processes. Int. J. Psychophysiol. 39, 241-248.

Bastiaansen, M., and Hagoort, P. (2006). Oscillatory neuronal dynamics during language comprehension. Prog. Brain Res. 159, 179-196.

Becker, R., Ritter, P., and Villringer, A. (2008). Influence of ongoing alpha rhythm on the visual evoked potential. Neuroimage 39, 707-716.

Bishop, G.H. (1933). Cyclic change in the excitability of the optic pathway of the rabbit. Am. J. Physiol. 103, 213-224.

Busch, N. A., Dubois, J., and VanRullen, R. (2009). The phase of ongoing EEG oscillations predicts visual perception. J. Neurosci. 29, 7869-7876.

Callaway, E. 3rd, and Yeager, C. L. (1960). Relationship between reaction time and electroencephalographic alpha phase. Science 132, 1765-1766.

Coles, G.H., and Rugg, M.D.(1995). “ERPs: an introduction," in Electrophysiology of Mind: Event Related Brain Potentials and Cognition, eds M. D. Rugg and G. H. Coles (Oxford: Oxford University Press), 16-17.

Crunelli, V., and Leresche, N. (1991). A role for $\mathrm{GABAB}$ receptors in excitation and inhibition of thalamocortical cells. Trends Neurosci. 14, 16-21.

Davidson, D. J., and Indefrey, P. (2007). An inverse relation between eventrelated and time-frequency violation responses in sentence processing. Brain Res. 1158, 81-92.

de Lange, F. P., Jensen, O., Bauer, M., and Toni, I. (2008). Interactions between posterior gamma and frontal alpha/ beta oscillations during imagined actions. Front. Hum. Neurosci. 2:7. doi: 10.3389/neuro.09.007.2008.

Fell, J., Dietl, T., Grunwald, T., Kurthen, M., Klaver, P., Trautner, P., Schaller, C., Elger, C. E., and Fernandez, G. (2004). Neural bases of cognitive ERPs: more than phase reset. J. Cogn. Neurosci. 16, 1595-1604.

Fell, J., Fernandez, G., Klaver, P., Elger, C. E., and Fries, P. (2003). Is synchronized neuronal gamma activity relevant for selective attention? Brain Res. Brain Res. Rev. 42, 265-272.

Fries, P., Nikolic, D., and Singer, W. (2007). The gamma cycle. Trends Neurosci. 30, 309-316.

Fukuda, K., Awh, E., and Vogel, E. K. (2010). Discrete capacity limits in visual working memory. Curr. Opin. Neurobiol. 20, 177-182.

Goldman, R. I., Stern, J.M., Engel, J. Jr., and and Cohen, M.S. (2002).Simultaneous EEG and fMRI of the alpha rhythm. Neuroreport 13, 2487-2492.

Goncalves, S. I., de Munck, J. C., Pouwels, P. J., Schoonhoven, R., Kuijer, J. P., Maurits, N. M., Hoogduin, J. M., Van Someren, E. J., Heethaar, R. M., and Lopes da Silva, F. H. (2006). Correlating the alpha rhythm to BOLD using simultaneous EEG/fMRI: inter-subject variability. Neuroimage 30, 203-213.

Gruber, W. R., Klimesch, W., Sauseng, P., and Doppelmayr, M. (2005). Alpha phase synchronization predicts $\mathrm{P} 1$ and N1 latency and amplitude size. Cereb. Cortex 15, 371-377.

Haegens, S., Osipova, D., Oostenveld, R., and Jensen, O. (2010). Somatosensory working memory performance in humans depends on both engagement and disengagement of regions in a distributed network. Hum. Brain Mapp. 31, 26-35.

Hagoort, P., and Brown, C.M. (2000).ERP effects of listening to speech compared to reading: the P600/SPS to syntactic violations in spoken sentences and rapid serial visual presentation. Neuropsychologia 38, 1531-1549.

Hamalainen, M. S., Hari, R., Ilmoniemi, R. J., Knuutila, J., and Lounasmaa. (1993). Magnetoencephalography. Theory, instrumentation and applications to the noninvasive study of brain function. Rev. Mod. Phys. 65, 413-497.

Hughes, S. W., and Crunelli, V. (2005). Thalamic mechanisms of EEG alpha rhythms and their pathological implications. Neuroscientist 11, 357-372.

Jensen, O., Gelfand, J., Kounios, J., and Lisman, J. E. (2002). Oscillations in the alpha band (9-12 Hz) increase with memory load during retention in a short-term memory task. Cereb. Cortex 12, 877-882.

Jensen, O., Kaiser, J., and Lachaux, J. P. (2007). Human gamma-frequency oscillations associated with attention and memory. Trends Neurosci. 30, 317-324.

Jensen, O., van Dijk, H., and Mazaheri, A. (2010). Amplitude asymmetry as a mechanism for the generation of slow evoked responses. Clin. Neurophysiol. 121, 1148-1149.

Jensen, O., and Mazaheri, A. (2010). Shaping functional architecture by oscillatory alpha activity: gating by inhibition. Front. Hum. Neurosci.4:186. doi:10.3389/fnhum.2010.00186

Jokisch, D., and Jensen, O. (2007) Modulation of gamma and alpha activity during a working memory task engaging the dorsal or ventral stream. J. Neurosci. 27, 3244-3251.

Jones, S. R., Pinto, D. J., Kaper, T. J., and Kopell, N. (2000). Alpha-frequency rhythms desynchronize over long cortical distances: a modeling study. J. Comput. Neurosci. 9, 271-291.

Kelly, S. P., Lalor, E. C., Reilly, R. B., and Foxe, J. J. (2006). Increases in alpha oscillatory power reflect an active retinotopic mechanism for distracter suppression during sustained visuospatial attention. J. Neurophysiol. 95, 3844-3851.

Kerlin, J. R., Shahin, A. J., and Miller, L. M. (2010). Attentional gain control of ongoing cortical speech representations in a "cocktail party". J. Neurosci. 30, 620-628.

Kilner, J. M., Vargas, C., Duval, S. Blakemore, S. J., and Sirigu, A. (2004). Motor activation prior to observation of a predicted movement. Nat. Neurosci. 7, 1299-1301.

Klimesch, W. (1999). EEG alpha and theta oscillations reflect cognitive and memory performance: a review and analysis. Brain Res. Brain Res. Rev. 29, 169-195.

Klimesch, W., Doppelmayr, M. Schimke, H., and Ripper, B. (1997). Theta synchronization and alpha desynchronization in a memory task. Psychophysiology 34, 169-176.

Klimesch, W., Doppelmayr, M., Schwaiger, J., Auinger, P., and Winkler, T. (1999). 'Paradoxical' alpha synchronization in a memory task. Brain Res. Cogn. Brain Res. 7, 493-501.

Klimesch, W., Sauseng, P., and Gruber, W. (2009). The functional relevance of phase reset: a comment to Risner et al. (2009): The visual evoked potential of surface alpha rhythm phase. Neuroimage 47, 5-7.

Klimesch, W., Sauseng, P., and Hanslmayr, S. (2007a). EEG alpha oscillations: the inhibition-timing hypothesis. Brain Res. Rev. 53, 63-88.

Klimesch, W., Sauseng, P., Hanslmayr, S., Gruber, W., and Freunberger, R. (2007b). Event-related phase reorganization may explain evoked neural dynamics. Neurosci. Biobehav. Rev. 31, 1003-1016.

Klimesch, W., Schack, B., Schabus, M., Doppelmayr, M., Gruber, W., and Sauseng, P. (2004). Phase-locked alpha and theta oscillations generate the $\mathrm{P} 1-\mathrm{N} 1$ complex and are related to memory performance. Brain Res. Cogn. Brain Res. 19, 302-316.

Klimesch, W., Schimke, H., Doppelmayr, M., Ripper, B., Schwaiger, J., and Pfurtscheller, G. (1996). Event-related desynchronization (ERD) and the Dm effect: does alpha desynchronization during encoding predict later recall performance? Int. J. Psychophysiol. 24, 47-60.

Kutas, M., and Hillyard, S. A. (1980). Reading senseless sentences: brain potentials reflect semantic incongruity. Science 207, 203-205.

Lachaux, J. P., George, N., Tallon-Baudry, C., Martinerie, J., Hugueville, L., Minotti, L., Kahane, P., and Renault, B. (2005). The many faces of the gamma band response to complex visual stimuli. Neuroimage 25, 491-501.

Lakatos, P., Chen, C. M., O'Connell, M. N., Mills, A., and Schroeder, C. E. (2007). Neuronal oscillations and multisensory interaction in primary auditory cortex. Neuron 53, 279-292. 
Lakatos, P., Karmos, G., Mehta, A. D., Ulbert, I., and Schroeder, C. E. (2008). Entrainment of neuronal oscillations as a mechanism of attentional selection. Science 320, 110-113.

Laufs, H., Holt, J. L., Elfont, R., Krams, M., Paul, J. S., Krakow, K., and Kleinschmidt, A. (2006). Where the BOLD signal goes when alpha EEG leaves. Neuroimage 31, 1408-1418.

Lehtela, L., Salmelin, R., and Hari, R. (1997). Evidence for reactive magnetic $10-\mathrm{Hz}$ rhythm in the human auditory cortex. Neurosci. Lett. 222, 111-114.

Lopes da Silva, F. H., van Rotterdam, A., Barts, P., van Heusden, E., and Burr, W. (1976). Models of neuronal populations: the basic mechanisms of rhythmicity. Prog. Brain Res. 45, 281-308.

Lorincz, M. L., Crunelli, V., and Hughes, S. W. (2008). Cellular dynamics of cholinergically induced alpha $(8-13 \mathrm{~Hz})$ rhythms in sensory thalamic nuclei in vitro. J. Neurosci. 28, 660-671.

Lorincz, M. L., Kekesi, K. A., Juhasz, G., Crunelli, V., and Hughes, S. W. (2009). Temporal framing of thalamic relaymode firing by phasic inhibition during the alpha rhythm. Neuron 63 , 683-696.

Makeig, S., Delorme, A., Westerfield, M., Jung, T. P., Townsend, J., Courchesne, E., and Sejnowski, T. J. (2004). Electroencephalographic brain dynamics following manually responded visual targets. PLoS Biol. 2, e176. doi: 10.1371/journal. pbio.0020176.

Makeig, S., Westerfield, M., Jung, T. P., Enghoff, S., Townsend, J., Courchesne, E., and Sejnowski, T.J.(2002). Dynamic brain sources of visual evoked responses. Science 295, 690-694.

Maki,H., and Ilmoniemi, R. J. (2010).EEG oscillations and magnetically evoked motor potentials reflect motor system excitability in overlapping neuronal populations. Clin. Neurophysiol. 121, 492-501.

Makinen, V., Tiitinen, H., and May, P. (2005). Auditory event-related responses are generated independently of ongoing brain activity. Neuroimage 24, 961-968.

Mathewson, K. E., Gratton, G., Fabiani, M., Beck, D. M., and Ro, T. (2009). To see or not to see: prestimulus alpha phase predicts visual awareness. $J$. Neurosci. 29, 2725-2732.

Mazaheri, A., and Jensen, O. (2006). Posterior alpha activity is not phasereset by visual stimuli. Proc. Natl. Acad. Sci. U.S.A. 103, 2948-2952.

Mazaheri, A., and Jensen, O. (2008). Asymmetric amplitude modulations of brain oscillations generate slow evoked responses. J. Neurosci. 28, 7781-7787.

Mazaheri, A., and Picton, T. W. (2005). EEG spectral dynamics during discrimination of auditory and visual targets. Brain Res. Cogn. Brain Res. 24, 81-96.

Medendorp, W. P., Kramer, G. F., Jensen, O., Oostenveld, R., Schoffelen, J. M., and Fries, P. (2007). Oscillatory activity in human parietal and occipital cortex shows hemispheric lateralization and memory effects in a delayed double-step saccade task. Cereb Cortex 17, 2364-2374.

Min, B. K., Busch, N. A., Debener, S., Kranczioch, C., Hanslmayr, S., Engel, A. K., and Herrmann, C. S. (2007). The best of both worlds: phase-reset of human EEG alpha activity and additive power contribute to ERP generation. Int. J. Psychophysiol. 65, 58-68.

Miniussi, C., and Thut, G. (2010). Combining TMS and EEG offers new prospects in cognitive neuroscience. Brain Topogr. 22, 249-256.

Murakami, S., and Okada, Y. (2006). Contributions of principal neocortical neurons to magnetoencephalography and electroencephalography signals. J. Physiol. (Lond.) 575(Pt 3), 925-936.

Nikulin, V. V., Linkenkaer-Hansen, K., Nolte, G., and Curio, G. (2010). Non-zero mean and asymmetry of neuronal oscillations have different implications for evoked responses. Clin. Neurophysiol. 121, 186-193.

Nikulin, V. V., Linkenkaer-Hansen, K., Nolte, G., Lemm, S., Müller K. R., Ilmoniemi R. J., and Curio, G. (2007). A novel mechanism for evoked responses in the human brain. Eur. J. Neurosci. 25, 3146-3154.

Osipova, D., Hermes, D., and Jensen, O. (2008). Gamma power is phaselocked to posterior alpha activity. PLoS ONE 3, e3990. doi: 10.1371/journal. pone. 0003990

Rihs, T. A., Michel, C. M., and Thut, G. (2007). Mechanisms of selective inhibition in visual spatial attention are indexed by alpha-band EEG synchronization. Eur. J. Neurosci. 25, 603-610.

Risner, M. L., Aura, C. J., Black, J. E., and Gawne, T.J. (2009). The visual evoked potential is independent of surface alpha rhythm phase. Neuroimage 45, 463-469.

Ritter, P., and Becker, R. (2009). Detecting alpha rhythm phase reset by phase sorting: caveats to consider. Neuroimage 47, 1-4.

Ritter, P., Moosmann, M., and Villringer, A. (2009). Rolandic alpha and beta EEG rhythms' strengths are inversely related to $\mathrm{AMRI-BOLD} \mathrm{signal} \mathrm{in} \mathrm{pri-}$ mary somatosensory and motor cortex. Hum. Brain Mapp. 30, 1168-1187.
Romei, V., Brodbeck, V., Michel, C. Amedi, A., Pascual-Leone, A., and Thut, G. (2007). Spontaneous fluctuations in posterior \{alpha\}-band EEG activity reflect variability in excitability of human visual areas. Cereb. Cortex. 18, 2010-2018.

Romei, V., Rihs, T., Brodbeck, V., and Thut, G. (2008). Resting electroencephalogram alpha-power over posterior sites indexes baseline visual cortex excitability. Neuroreport 19 203-208.

Rugg, M. D., and Curran, T. (2007). Event-related potentials and recognition memory. Trends Cogn. Sci. (Regul. Ed.) 11, 251-257.

Salenius, S., Schnitzler, A., Salmelin, R. Jousmaki, V., and Hari, R. (1997). Modulation of human cortical rolandic rhythms during natural sensorimotor tasks. Neuroimage 5, 221-228.

Sanquist, T. F., Rohrbaugh, J. W. Syndulko, K., and Lindsley, D. B. (1980). Electrocortical signs of levels of processing: perceptual analysis and recognition memory. Psychophysiology 17, 568-576.

Sauseng, P., Klimesch, W., Freunberger, R. Pecherstorfer, T., Hanslmayr, S., and Doppelmayr, M. (2006). Relevance of EEG alpha and theta oscillations during task switching. Exp. Brain Res. 170, 295-301.

Sauseng, P., Klimesch, W., Heise, K. F., Gruber, W. R., Holz, E., Karim, A. A., Glennon, M., Gerloff, C., Birbaumer, N., and Hummel, F. C. (2009). Brain oscillatory substrates of visual shortterm memory capacity. Curr. Biol. 19, 1846-1852.

Scheeringa, R., Petersson, K. M., Oostenveld, R., Norris, D. G., Hagoort, P., and Bastiaansen, M. C. (2009). Trialby-trial coupling between EEG and BOLD identifies networks related to alpha and theta EEG power increases during working memory maintenance. Neuroimage 44, 1224-1238.

Schroeder, C. E., and Lakatos, P. (2009). Low-frequency neuronal oscillations as instruments of sensory selection. Trends Neurosci. 32, 9-18.

Shah, A. S., Bressler, S. L., Knuth, K. H., Ding, M., Mehta, A. D., Ulbert, I., and Schroeder, C. E. (2004). Neural dynamics and the fundamental mechanisms of event-related brain potentials. Cereb. Cortex 14, 476-483.

Shahin, A. J., Picton, T. W., and Miller, L. M. (2009). Brain oscillations during semantic evaluation of speech. Brain Cogn. 70, 259-266.

Soltani, M., and Knight, R. T. (2000) Neural origins of the P300. Crit. Rev. Neurobiol. 14, 199-224.

Stam, C. J., Pijn, J. P., Suffczynski, P., and Lopes da Silva, F. H. (1999a). Dynamics of the human alpha rhythm: evidence for non-linearity? Clin. Neurophysiol. 110, 1801-1813.

Stam, C. J., Vliegen, J. H., and Nicolai, J. (1999b). Investigation of the dynamics underlying periodic complexes in the EEG. Biol. Cybern. 80, 57-69.

Takashima, A., Jensen, O., Oostenveld, R., Maris, E., van de Coevering, M., and Fernandez, G. (2006). Successful declarative memory formation is associated with ongoing activity during encoding in a distributed neocortical network related to working memory: a magnetoencephalography study. Neuroscience 139 , 291-297.

Thut, G., and Miniussi, C. (2009). New insights into rhythmic brain activity from TMS-EEG studies. Trends Cogn. Sci. (Regul. Ed.) 13, 182-189.

Thut, G., Nietzel, A., Brandt, S. A., and Pascual-Leone, A. (2006). Alpha-band electroencephalographic activity over occipital cortex indexes visuospatial attention bias and predicts visual target detection. J. Neurosci. 26, 9494-9502.

Thut, G., Theoret, H., Pfennig, A., Ives, J., Kampmann, F., Northoff, G., and Pascual-Leone, A. (2003). Differential effects of low-frequency rTMS at the occipital pole on visual-induced alpha desynchronization and visual-evoked potentials. Neuroimage 18, 334-347.

Tuladhar, A. M., ter Huurne, N., Schoffelen, J.M., Maris, E., Oostenveld, R., and Jensen, O. (2007). Parietooccipital sources account for the increase in alpha activity with working memory load. Hum. Brain Mapp. 28, 785-792.

van Dijk, H., Schoffelen, J. M., Oostenveld, R., and Jensen, O. (2008). Prestimulus oscillatory activity in the alpha band predicts visual discrimination ability. J. Neurosci. 28, 1816-1823.

van Dijk, H., Nieuwenhuis, I. L., and Jensen, O. (2010a). Left temporal alpha band activity increases during working memory retention of pitches. Eur. J. Neurosci. 31, 1701-1707.

van Dijk, H., van der Werf, J., Mazaheri, A., Medendorp, W. P., and Jensen, O. (2010b). Modulations in oscillatory activity with amplitude asymmetry can produce cognitively relevant event-related responses. Proc. Natl. Acad. Sci. U.S.A. 107, 900-905

van Elswijk, G., Maij, F., Schoffelen, J. M., Overeem, S., Stegeman, D. F., and Fries, P. (2010). Corticospinal betaband synchronization entails rhythmic gain modulation. J. Neurosci. 30, 4481-4488.

VanRullen, R., and Koch, C. (2003). Is perception discrete or continuous? Trends Cogn. Sci. 7, 207-213. 
Varela, F. J., Toro, A., John, E. R., and Schwartz, E. L. (1981). Perceptual framing and cortical alpha rhythm. Neuropsychologia 19, 675-686.

Vogel, E. K., and Machizawa, M. G. (2004). Neural activity predicts individual differences in visual working memory capacity. Nature 428, 748-751.

Vogel, E. K., McCollough, A. W., and Machizawa, M. G. (2005). Neural measures reveal individual differences in controlling access to working memory. Nature 438, 500-503.

Walter, W. G., Cooper, R., Aldridge, V. J., McCallum, W. C., and Winter, A. L. (1964). Contingent negative variation: an electric sign of sensorimotor association and expect- ancy in the human brain. Nature 203, 380-384.

Womelsdorf, T., Fries, P., Mitra, P. P., and Desimone, R. (2006). Gamma-band synchronization in visual cortex predicts speed of change detection. Nature 439, 733-736.

Worden, M. S., Foxe, J. J., Wang, N., and Simpson, G. V. (2000). Anticipatory biasing of visuospatial attention indexed by retinotopically specific alpha-band electroencephalography increases over occipital cortex. J. Neurosci. 20, RC63.

Wu, J., and Okada, Y. C. (1999). Roles of a potassium afterhyperpolarization current in generating neuromagnetic fields and field potentials in longitudinal CA3 slices of the guinea-pig. Clin. Neurophysiol. 110, 1858-1867.
Yuan, H., Liu, T., Szarkowski, R., Rios, C., Ashe, J., and He, B. (2010). Negative covariation between task-related responses in alpha/beta-band activity and BOLD in human sensorimotor cortex: an EEG and fMRI study of motor imagery and movements. Neuroimage 49, 2596-2606.

Zhang, Y., Wang, X., Bressler, S. L., Chen, Y., and Ding, M. (2008). Prestimulus cortical activity is correlated with speed of visuomotor processing. J. Cogn. Neurosci. 20, 1915-1925.

Conflict of Interest Statement: The authors declare that the research was conducted in the absence of any commercial or financial relationships that could be construed as a potential conflict of interest.

Received: 21 May 2010; paper pending published: 12 June 2010; accepted: 25 August 2010; published online: 14 October 2010.

Citation: Mazaheri $A$ and Jensen $O$ (2010) Rhythmic pulsing: linking ongoing brain activity with evoked responses. Front. Hum. Neurosci. 4:177. doi: 10.3389/ fnhum.2010.00177

Copyright (C) 2010 Mazaheri and Jensen. This is an open-access article subject to an exclusive license agreement between the authors and the Frontiers Research Foundation, which permits unrestricted use, distribution, and reproduction in any medium, provided the original authors and source are credited. 